\title{
Multi-Scale Modeling Of Materials: A Basis for Computational Design
}

\author{
$\underline{\text { I.S.Cole }}^{1}$, C. Chu ${ }^{1}$, M.Breedon ${ }^{1}$, D.Winkler ${ }^{1}$ \\ ${ }^{1}$ CSIRO Materials Science and Engineering, Private bag 33, Clayton South MDC, Victoria 3169, Australia \\ Email:ivan.cole@csiro.au
}

\begin{abstract}
This paper will present a multi-scale of corrosion that spans scales from molecular scale (where it considers the binding of inhibitor to metal surfaces) to the continental scale where it considers ocean production of marine aerosols and the aerosols subsequent transport across continents. The multi-scale model has been developed in order to both guide the development of new corrosion resistant materials and to aid in their design and selection. Multi-scale modelling will facilitate computational assisted design by allowing the effect of a large number of design variables to be assessed computationally before the final reduced selection can be investigated experimentally.

The combination of macro - micron scale allows a definition of the state of the surface (on a 3 hourly basis) with four states being defined: wet from rain, wet from the wetting of hygroscopic salts, drying, and dry. Then for each these states the rate of corrosion and changes in the nature of the metal/oxide/moisture layer system are predicted. On active metals such as zinc and steel, multi-layers of oxide may develop. Adjacent to the metal surface a compact oxide is observed under certain circumstances. This compact oxide layer dramatically reduces the rate of metallic corrosion. Above, or in the absence of the compact layer, bands of porous oxide may develop with variable porosity from the metal or compact layer interface to the moisture layer. To model this porous oxide a model based on porous-electrode theory has been developed. The porous oxide model (POM) is a continium model that accounts for diffusion, chemical and electrochemical process. In a system where the oxide is semi-conducting (such as zinc or iron) the oxide itself may support the oxygen reduction reaction (ORR). The POM considers the relative rate of the ORR at the metal surface and at the porous oxide-solution boundary. It is found that the ORR occurs both at the metal surface and at the pore boundary but with time as oxygen is depleted at the bottom of the pores the ORR occurs predominantly on the oxide surface. The conditions generated within the porous layer will affect the conditions at the porous layer/compact layer interface and thus affect the compact layer stability. The POM model is then combined with a fine scale model of the condition of and processes within compact barrier oxides. The integration of the state model the POM and the model of the compact oxide will provide both a design and materials selection tool for uncoated metals
\end{abstract}

Modelling of inhibitor/metal surface interaction is being undertaken to assess the effect of molecular structure and functional groups that lead to effective inhibition. This model combines a range of submodules, including modules which cover extensive length scales.

Keywords: Corrosion, Inhibitor, Coating, Oxide, Multi-scale Modeling 


\section{INTRODUCTION}

A major challenge for the development of new materials for engineered structures is the time required to take a new material from an idea to a commercial product is significantly in excess of that required to design and build a new engineered structure. For example the time for the development of a new aircraft is currently 6-8 years, while that of to develop a new aerospace material may be up to 15 years (Osborne et al., 2001). This renders the process of getting new materials onto aircraft very difficult as materials must be planned a generation ahead of air-platforms, and clearly specifications may change for those platforms. Thus, there is a significant drive to dramatically reduce the time for the development and certification of materials. Materials development time may be divided into the discovery phase, the research and development phase, scale up, certification and testing and then production optimization. The use of tools such as computational design has the potential to significantly reduce the discovery and research and development phase for new materials (Le, et.al, 2012).

Computational design harness models across a wide range of scales so that the effect of design changes of critical elements of a material of the function and life of full scale components in service can be determined. Consider the computational design of inhibited paints for protection of the fuselage of aircraft. Traditionally chromates (particularly strontium chromate) have been added to primers to provide ongoing active inhibition against metal corrosion (Kendig \& Buchheit, 2003). The chromate will leach out of the primer and migrate to any exposed metal surfaces (bottom of cracks) where it can bind to the surface reducing the probability of corrosion. Unfortunately chromate is a human toxin and is being withdrawn from service so new safe inhibitors are required (Costa \& Klein, 2006). Two significant groups are of interests, the rare earths (Hughes, et al., 2010) (cerium, lanthanum, praseodymium) and organic heterocyclic structures (Harvey et al., 2011). The heterocyclic compounds can be effective anodic inhibitions while their efficiency depends critically on what functional groups are attached to the ring structures, where on the ring the groups are attached (Harvey et al., 2011), and how electrons are redistributed once the molecule has absorbed onto a surface. The rare earth elements can act as cathodic inhibitors, by promoting the development of mixed oxides on cathodic sites. Thus, the effectiveness of inhibitors in coatings will depend on the ability of the inhibitor to bond to the surface (which is controlled by its molecular structure in the case of organic inhibitors) and the ability of the coating system to deliver a sufficient concentration of inhibitor to defects in its structure (this will depend on the concentration of inhibitor in the film, the release rate from the film, the diffusion rate through the film, and through any moisture layers). The total life of an inhibited system will further depend on the pit initiation and growth rate (given the inhibitor concentration at defects), the nature and frequency and of surface wetting and the microclimate, macroclimate and usage patterns that control such wetting. Thus a multi-scale model to permit the design of inhibited paint films would have to span scales from $10^{-10} \mathrm{~m}$ to $10^{6} \mathrm{~m}$. The former limit being the scale of interactions between functional groups on a molecule and a surface, and the later being the scale required to predict pollutant (particularly marine aerosol) formation and transport (which controls surface wetting). At present a range of multi-scale corrosion models have been developed (I. S. Cole, 2002) and while some cover an impressive range of scales (I. S. Cole, et al. 2011; I. S. Cole, et al. 2003), from around $10^{-6} \mathrm{~m}$ to $10^{6} \mathrm{~m}$ this range must be extended to permit computational design.

A second major advantage of computational design is that it can provide reasonable estimate of the probable effective life of material systems. The prediction of the service life of traditional inhibited coatings is based on a combination of experience and performance in accelerated environmental tests; such tests are used to determine if the quality of the coatings matches those currently in service. The life of coatings in service is often estimated from experience, unfortunately there is no long-term experience with new "green" inhibitors. If designers and regulators are going to have confidence in structures protected by green inhibitors, then an accurate life predictive method is required. A rigorously qualified computational design method may be able to meet this requirement. This paper will outline the development of computational design and multi-scale modelling of inhibited coatings applied to airframes. It will highlight the advantages of building an adaptable system based on a flexible platform that can integrate models, data and information across a range of scales. It will explore the lessons learned in developing such a system and highlight limitations and challenges to be addressed. To the authors knowledge the model being developed is the first in the corrosion field to spans from the molecular to macro-climate scales and so the first model that can allow design of the molecular structure of inhibitors for final in film service performance. Other work only looks at sub-systems molecular to electrochemical (Gece, 2008) or electrochemical to pit growth (Xiao \& Chaudhuri, 2011). 


\section{MULTI-SCALE FRAMEWORK}

A system framework has been designed to link together models across the scales from $10^{-10} \mathrm{~m}$ to $10^{6} \mathrm{~m}$ in a dynamic manner via the hierarchal linking/interconnection of these modules starting from the smallest length scale, and sequentially proceeding to the largest scale of interest. The model framework also permits incorporation of experimental data and subsequent modification of such data and upgrading of models (e.g QSAR models) partially based on this data. The architecture, as presented in Figure 1, is divided into two segments to increase the framework extensibility to achieve effective deployment of the different models. Flexibility is an important aspect of the design to support multiple model components which have been developed in different programming languages. To facilitate this, plug-ins have been employed to map the invocation of a model to the corresponding input and output data, with each model considered as a plug-in. A plug-in includes the model executable program and an XML document. The XML document is in plain text format and details all the model information which will be initialized by the plug-in manager so that it may be represented on the front-end viewer. Based on the plug-in architecture, the framework is able to recognize a new model without modifying the framework that supports it. Since the framework is in Java, the application is able to run on multiple platforms. User interface scalability is also a concern in designing the framework to support multiple models. The workflow concept is applied to represent the relationship of all models. It allows models to be linked up together step-by-step to make the design straightforward and meaningful. The framework allows users to use it as a computational tool, while providing them with a workflow editor to investigate and design a new model.

All models are interconnected by linking the output of the first model with the input of the next model. In order to establish the connection, both the data type of the input and output data must be consistent. Since the system is already defined by the information contained within model XML document, the interface is designed to be user-friendly and straightforward, by linking two models together via a drag-and-drop type visual development tool. The system also provides the validation for checking each input-output data type to confirm the consistency in order to reduce the runtime errors.

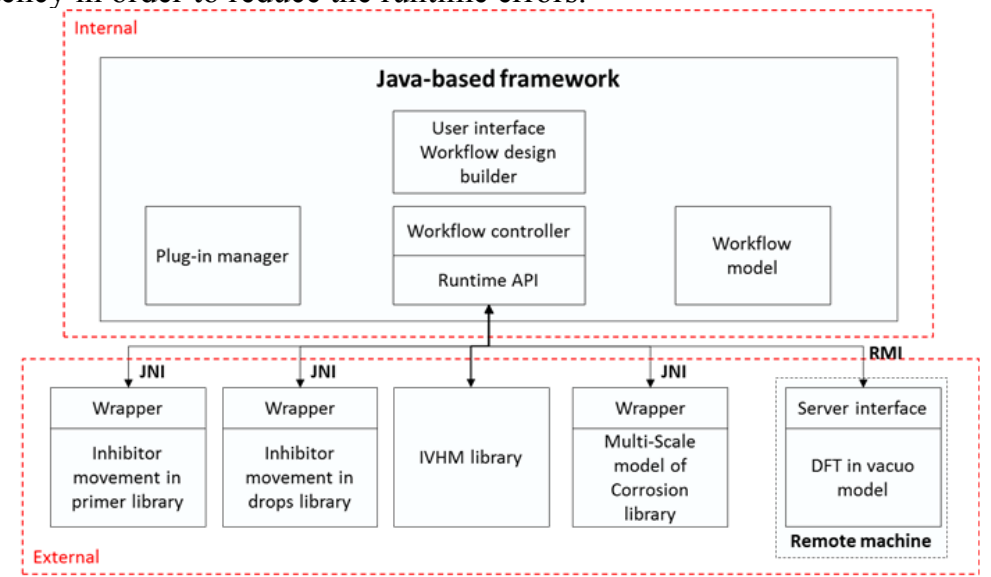

Figure 1. Framework Architecture (JNI: Java native interface, RMI: remote method invocation)

The system controls data flow through the workflow model structure. The diagram in Figure 2 illustrates how to manage the data within the system. All input and output data transmission between modules would go through the system; therefore the system can perform an optimization/task after receiving data from one module and before sending data to another module. The optimization can include filtering data under some condition, converting data type from one to another or mapping data with designated data table.

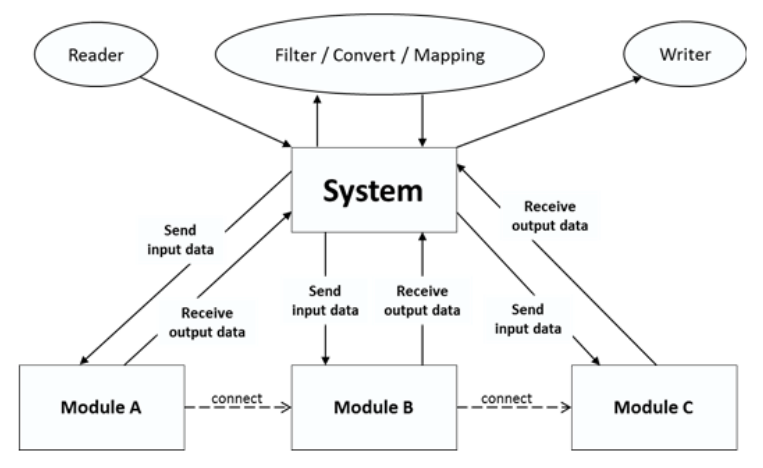

Figure 2. Data Flow Diagram 
To provide the capability outlined in the introduction modules have been developed to

A) Estimate the molecular properties of inhibitors

B) Develop relationships between molecular properties and inhibitor efficiency or electrochemical properties

C) Calculate the diffusion of a inhibitor from through a film to a defect

D) Calculate the pitting rate of an exposed metal surface

E) Calculate when corrosion can occur

F) Sum up the corrosion events and predict the life of a component

Due to space limitations, this paper will concentrate on Modules A, B and C as they illustrate interesting points in terms of data use and model construction.

\section{MOLECULAR TO ELECTROCHEMICAL MODELLING}

To calculate molecular properties which may be relevant to corrosion inhibition efficacy, density functional theory (DFT) was employed. As a proof of concept, we have created a module which links a supercomputer hosted version of SIESTA (Soler et al., 2002) (a DFT code) to the multi-scale design framework, handling molecular model generation and data parsing/communication through to results analysis. Data which is parsed to the next module contains the corrosion inhibitor's molecular structure and properties (numerical value, such as ionization potential energy) of the investigated corrosion inhibitor molecule. The molecular properties which were considered in the model include: electron affinity, ionization potential, fundamental bandgap, Mulliken electronegativity, chemical hardness and chemical potential. The specifics of the SIESTA calculations are as follows: the exchange correlation functional of Perdew-Burke-Ernzerhof within the generalized gradient approximation (GGA) with a cut off energy of 500 Ry employed throughout. A double zeta plus polarization (DZP) basis set was used for all calculations, and norm-conserving pseudopotentials were used as supplied with the SIESTA code without further modification. Geometry optimisation was performed with the conjugate gradient (CG) algorithm until the residual forces on atoms were less than $0.01 \mathrm{eV} / \AA$, and the difference in total energy between SCF steps was less than $10^{-4} \mathrm{eV}$. Potential corrosion inhibitor molecules were modeled by centering a molecule into a fixed $30 \AA^{3}$ supercell using the $\Gamma$-point only, and allowing full structural relaxation.

This model loads as a self contained module with its own GUI driven wizard. The wizard starts with the selection of molecule, which can be manually inputted by the user or dynamically sourced from rich cloud sources and cached propriety databases. This section of the wizard also features an embedded visualisation applet, permitting visual confirmation of selected molecule. The user then selects the remote machine details and a communication test is executed before selecting the job script parameters; instructing the supercomputer on how to run the job. The wizard prepares the job locally by writing out SIESTA input files and downloading the pertinent pseudopotentials from the SIESTA servers, which is then collated with the input data in a locally stored job folder. This folder is then uploaded to the supercomputer via an SSH protocol and remotely executed. This step removes human error associated with manual data entry, particularly the incorrect formatting of the initial molecular geometry. The job is now placed in the remote supercomputer queue awaiting execution. This brings the user to the final stage of the wizard, where the job progress, results, raw output, and job summary is presented; along with any other "molecular job" which have been submitted through the wizard, as depicted in Figure 3. These calculations require the output (atomic positions) of the first job to be used as the input for the single point calculations required for electron affinity and ionization potential determination as depicted in Figure 4.

This wizard based system could be adapted to automate searching of cloud hosted molecular databases for molecular features which the user believes would be conducive to excellent corrosion inhibition efficacy. The automatic calculation of such molecules would permit the user, presumably a scientist/engineer to focus on the analysis of the data, rather than in time consuming data entry and job management tasks. In addition to molecular properties generated from DFT calculations, other properties or descriptors (e.g. number of sulfur atoms, number of ionized sulfur atoms, number of ionized $\mathrm{COOH}$ groups, number of rings, number of hetero-aromatic nitrogen groups, total molecular charge) are derived using the DRAGON program (Todeschini \& Consonni, 2000) and our in-house modeling package, BioModeller (Burden, et al., 2009; Winkler \& Burden, 2000; Winkler, et al. 1998). All the molecular properties and descriptors are then combined with experimental data on inhibitor performance and run through a quantitative structure property relationship (QSPR) module (BioModeller) Experimental data can take various forms such as measured inhibitor efficiency or particular electrochemical parameters. The QSAR module uses machine learning methods to build relationships between molecular properties and experimental values related to corrosion 


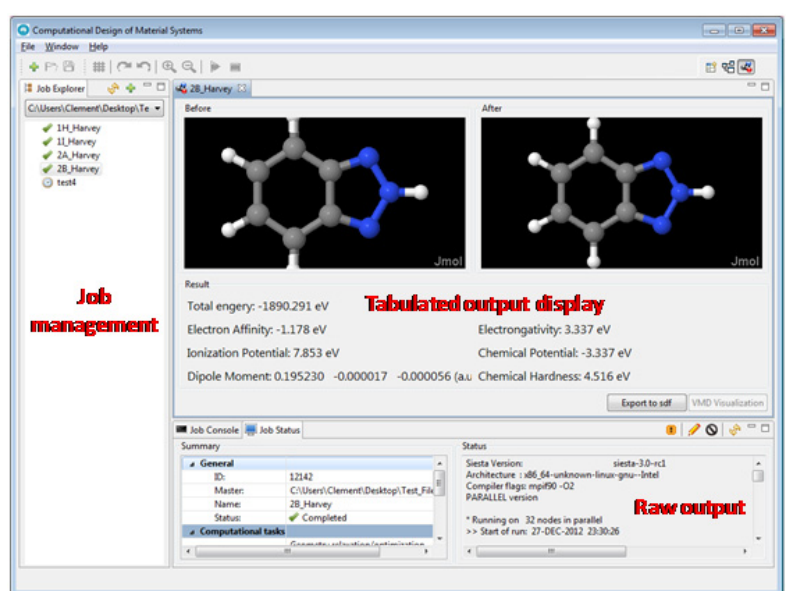

Figure 3. Job management and output screen of the molecular modeling module.

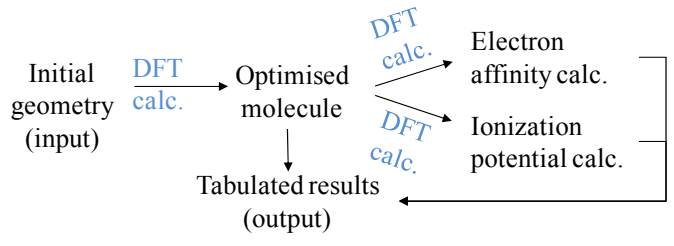

Figure 4. Flow diagram of the steps required for generating the tabulated results; here DFT calc. represents a step where the module prepares a job, communicates it to the supercomputer, executes the code remotely, and downloads the results automatically once the supercomputer task has completed.

inhibitor and then in future application of the framework these relationships are used to predict

electrochemical properties from molecular properties and descriptors. Both linear and nonlinear relationships (models) were generated. Linear models were sparse linear regression calculations. The nonlinear models using a Bayesian regularized neural network (Burden et al., 2009; Burden \& Winkler, 2009a) that automatically controls model complexity to optimize the predictive performance of the models. The neural network was trained to the maximum in the Bayesian evidence for the models. Generally two or three hidden layer nodes were employed in a three layer neural network. The input and output layers nodes contained linear transfer functions, and the hidden layer nodes (where the computation is carried out) employed nonlinear sigmoidal transfer functions. The performance of the models was assessed using the standard error of the prediction of the training and test sets (Figure 5).

We used the sparse feature selection capabilities of BioModeller to select the most relevant subset of descriptors from a large pool, in a context dependent manner. We generated statistically valid models that could predict the corrosion inhibition of compounds in an external test set using DRAGON descriptors and in-house chemically intuitive descriptors. We found a set of between 7 and 9 descriptors in each descriptor family could generate linear and nonlinear models that could make good, quantitative predictions of the degree of inhibition for the data set. The models obtained using descriptors based on the speciated form of the inhibitors at $\mathrm{pH} 7$ were similar to those when neutral inhibitors were assumed, so the results for the neutral form of the molecules are reported here.
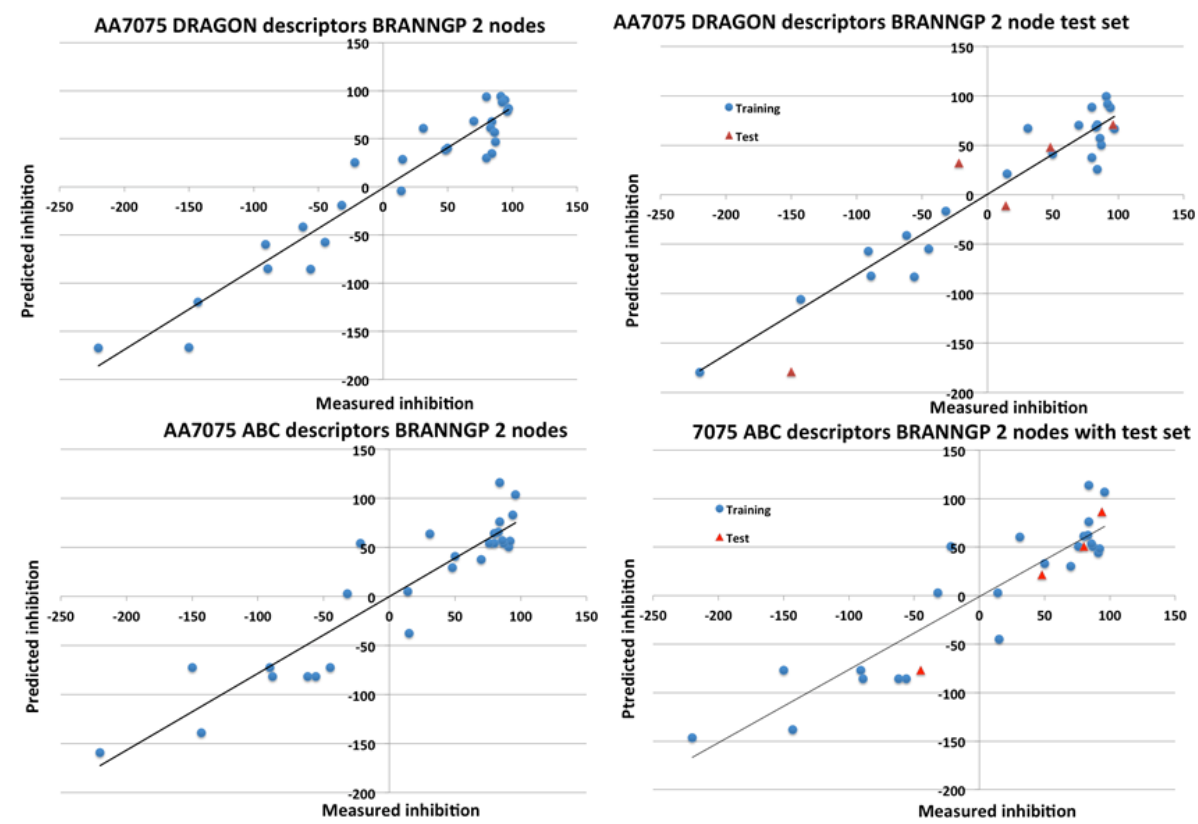

Figure 5. The observed versus predicted corrosion inhibition for 7075 alloy. Left panel shows models for entire data set of 28 compounds and right panel shows data set split into training (circles) and test (triangles) sets. The top graphs shows the nonlinear model using DRAGON descriptors, and the bottom graphs give the performance of the nonlinear models using the in-house intuitive descriptors. 


\section{CORROSION, AND MACRO SCALES CONTROLLING FACTORS PROMOTING CORROSION}

Corrosion can occur if the surface is wet and two modules have been developed to predict when such moisture will occur on surfaces: one module is applied to the exterior of a plan and the other to the interior. In both models the user defines a usage pattern of the aircraft, which provides flight schedules and particulars concerning flight patterns, speeds, storage on ground etc. The exterior approach uses processes models (Hughes et al., 2007) and meteorological data to estimate likely moisture events based on past meteorological information for flight locations, as exterior relative humidity is very low above the cloud level formation of wetness layers on surfaces in flights will not occur and so wetness events need only be calculated when the aircraft is on ground although pollutants may deposit in flight (I. S. Cole \& Paterson, 2009). Models include analytical models of aerosol formation and computer fluid dynamic models of aerosol transport and deposition onto aircraft (I. S. Cole \& Paterson, 2009), analytical and heat and mass transfer models for prediction surface temperatures and evaporation and condensation (I. S. Cole \& Paterson, 2006) and computer fluid dynamics and analytical models for predicting drop movement on a surface and retention of contaminates on a surface (I. S. Cole \& Paterson, 2007). The approach for predicting wetness on surfaces within an airframe is quite different (Trego et al., 2007). Wetness events on surfaces within an aircraft will often occur because of human intervention, either directly (spilling a drink, etc.) or indirectly (failure to replace an insulation blanket that leads to condensation) so that it is difficult if not impossible to predict such events from process models (I. S. Cole et al., 2012).

Rather semi-empirical models are developed from direct measurements of wetness and microclimate on aircraft in flight. Two cases are identified, where wetness events are explicable in terms of standard thermodynamics of moist air (condensation, evaporation) and where they are not and appear associated with human activity (spills in the galley or lavatory etc). In the later case a probabilistic model of the occurrence of moisture is developed directly from direct measurement of the frequency of human induced moisture events. In the former case the microclimate data is used to develop two empirical models, one of the rate of air exchange between the interior environment of the sub-floor and the air exterior to the aircraft and the other of the rate of change in temperature of surfaces in the subfloor of an aircraft after landing. These two models are combined with meteorological data defining air conditions on ground and in flight to predict the probability of condensation and evaporation as the aircraft flies between bases around the globe (I.S. Cole, et al., 2013).

\section{DISCUSSION}

Although the modeling effort is not yet completed, it does indeed look like the sequence of models linked together will be able to predict the life (at least to a reasonable approximation) of an inhibited coating in actual service. Once verified this will be an important conclusion as it will open the possibility of design based on multi-scale modeling. The corollary of this conclusion is that the demand on scientist's time to construct such a series of linked model is very high. This activity is likely to be successful as it adopts an open framework that can call on models developed by a number of groups in prior programs prior to the computational design effort. Collaboration and open frameworks will be necessary if computational design is to be developed in reasonable times. The model system outlined combines a number of sources and types of information, information from experimental and monitoring programs, information extracted from on-line data sources and tools and information calculated from in-house programs. It is very unlikely that a system of in-house models will be able cover the scales required, not just because of man hours required, but because the theoretical frameworks connecting different models (e.g. molecular and electrochemical) are not sufficiently established, or in the case of aircraft microclimate the variability and uncertainty in parameters is too high. In these cases approaches based on data (such as the use of QSAR to connect molecular properties and electrochemical parameters) are required. However data based approaches also offer the advantage that as the data is enriched, defined relationships between outputs and inputs can be refined so the framework can develop.

\section{CONCLUSION}

This paper has outlined the development of a multi-scale model for predicting the life of paint films containing active inhibitors. The paper illustrates that such models can be developed but will require

1) Open frameworks to permit the integration of models and information of different types and from different sources

2) The inclusion of data and data derived models will be necessary in such models for the foreseeable future due to conceptual and theoretical gaps between scales and processes. However such data 
derived approaches also offer the possibility of adapting the approach when additional data is available.

\section{REFERENCES}

Burden, F. R., Polley, M. J., \& Winkler, D. A. (2009). Toward Novel Universal Descriptors: Charge Fingerprints. Journal of Chemical Information and Modeling, 49(3), 710-715.

Burden, F. R., \& Winkler, D. A. (2009a). Optimal Sparse Descriptor Selection for QSAR Using Bayesian Methods. Qsar \& Combinatorial Science, 28(6-7), 645-653.

Cole, I. S. (2002). Recent progress in modelling atmospheric corrosion. Corrosion Reviews, 20(4-5), 317337.

Cole, I. S., Ganther, W. G., Corrigan, P., Galea, S., Trathen, P., \& Hinton, B. (2012). Frequency and duration of wetness periods on surfaces in airframes. Corrosion Engineering Science and Technology, 47(7), 529-535.

Cole, I. S., Muster, T. H., Azmat, N. S., Venkatraman, M. S., \& Cook, A. (2011). Multiscale modelling of the corrosion of metals under atmospheric corrosion. Electrochimica Acta, 56(4), 1856-1865.

Cole, I. S., \& Paterson, D. A. (2006). Mathematical models of dependence of surface temperatures of exposed metal plates on environmental parameters. Corrosion Engineering Science and Technology, 41(1), 67-76.

Cole, I. S., \& Paterson, D. A. (2007). Holistic model for atmospheric corrosion - Part 7 - Cleaning of salt from metal surfaces. Corrosion Engineering Science and Technology, 42(2), 106-111.

Cole, I. S., \& Paterson, D. A. (2009). Modelling aerosol deposition rates on aircraft and implications for pollutant accumulation and corrosion. Corrosion Engineering Science and Technology, 44(5), 332339.

Cole, I.S., Corrigan, P., Ganther, W., \& Galea, S. (2013). Recent Progress in intelligent vehichle health monitoring Key Engineering materials 558, 357-363.

Costa, M., \& Klein, C. B. (2006). Toxicity and carcinogenicity of chromium compounds in humans. Critical Reviews in Toxicology, 36(2), 155-163.

Gece, G. (2008). The use of quantum chemical methods in corrosion inhibitor studies. Corrosion Science, 50(11), 2981-2992.

Harvey, T. G., Hardin, S. G., Hughes, A. E., Muster, T. H., White, P. A., Markley, T. A., Glenn, A. M. (2011). The effect of inhibitor structure on the corrosion of AA2024 and AA7075. Corrosion Science, 53(6), 2184-2190.

Hughes, A. E., Cole, I. S., Muster, T. H., \& Varley, R. J. (2010). Designing green, self-healing coatings for metal protection. Npg Asia Materials, 2(4), 143-151.

Hughes, A. E., Hinton, B., Furman, S. A., Cole, I. S., Paterson, D., Stonham, A., Ranson, M. (2007). Airlife Towards a fleet management tool for corrosion damage. Corrosion Reviews, 25(3-4), 275-293.

Kendig, M. W., \& Buchheit, R. G. (2003). Corrosion inhibition of aluminum and aluminum alloys by soluble chromates, chromate coatings, and chromate-free coatings. Corrosion, 59(5), 379-400.

Le, Tu, Epa, V. Chandana, Burden, Frank R., \& Winkler, David A. (2012). Quantitative Structure-Property Relationship Modeling of Diverse Materials Properties. Chemical Reviews, 112(5), 2889-2919. doi: $10.1021 / \mathrm{cr} 200066 \mathrm{~h}$

Osborne, J. H., Blohowiak, K. Y., Taylor, S. R., Hunter, C., Bierwagon, G., Carlson, B., . . Donley, M. S. (2001). Testing and evaluation of nonchromated coating systems for aerospace applications. Progress in Organic Coatings, 41(4), 217-225.

Soler, J. M., Artacho, E., Gale, J. D., García, A., Junquera, J., Ordejón, P., \& Sánchez-Portal, D. (2002). The SIESTA method for ab initio order-N materials simulation. Journal of Physics Condensed Matter, 14(11), 2745-2779.

Todeschini, R., \& Consonni, V. (2000). Handbook of Molecular Descriptors. Weinheim: Wiley-VCH.

Trego, A., Price, D., Hedley, M., Corrigan, P., Cole, I., \& Muster, T. (2007). Development of a system for corrosion diagnostics and prognostics. Corrosion Reviews, 25(1-2), 161-177.

Winkler, D. A., \& Burden, F. R. (2000). Robust QSAR models from novel descriptors and Bayesian Regularised Neural Networks. Molecular Simulation, 24(4-6), 243-258.

Winkler, D. A., Burden, F. R., \& Watkins, A. J. R. (1998). Atomistic topological indices applied to benzodiazepines using various regression methods. Quantitative Structure-Activity Relationships, 17(1), 14-19.

Xiao, J., \& Chaudhuri, S. (2011). Predictive modeling of localized corrosion: An application to aluminum alloys. Electrochimica Acta, 56(16), 5630-5641. 